\title{
Circular dichroism of magneto-phonon resonance in doped graphene
}

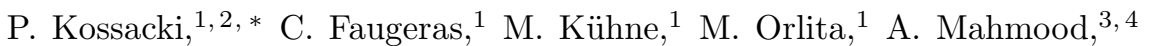 \\ E. Dujardin, ${ }^{5}$ R.R. Nair, ${ }^{6}$ A.K. Geim, ${ }^{6}$ and M. Potemski ${ }^{1}$ \\ ${ }^{1}$ Laboratoire National des Champs Magneétiques Intenses, \\ CNRS/UJF/UPS/INSA, F-38042 Grenoble, France \\ ${ }^{2}$ Institute of Experimental Physics, University of Warsaw, Hoza 69, 00-681 Warsaw, Poland \\ ${ }^{3}$ CEMES-CNRS, Universitè de Toulouse, 29 rue Jeanne Marvig, 31055 Toulouse, France \\ ${ }^{4}$ CNRS-Institut Néel, BP 166, 38042 Grenoble Cedex 9, France \\ ${ }^{5}$ CEMES-CNRS, Université de Toulouse, 29 rue Jeanne Marvig, 31055 Toulouse, France \\ ${ }^{6}$ School of Physics and Astronomy and Centre for Mesoscience and Nanotechnology, \\ University of Manchester, Manchester M13 9PL, UK
}

(Dated: July 9, 2018)

\begin{abstract}
Polarization resolved, Raman scattering response due to $\mathrm{E}_{2 g}$ phonons in monolayer graphene has been investigated in magnetic fields up to $29 \mathrm{~T}$. The hybridization of the $\mathrm{E}_{2 g}$ phonon is only observed with the fundamental inter Landau level excitation (involving the $\mathrm{n}=0$ Landau level) and in just one of the two configurations of the circularly crossed polarized excitation and scattered light. This polarization anisotropy of the magneto-phonon resonance is shown to be inherent to relatively strongly doped graphene samples, with carrier concentrations typical for graphene deposited on $\mathrm{Si} / \mathrm{SiO}_{2}$ substrates.

PACS numbers: 73.22.Lp, 63.20.Kd, 78.30.Na, 78.67.-n
\end{abstract}

The effective coupling of optical phonons $\left(\mathrm{E}_{2 g}\right)$ to electronic excitations in graphene yields a particularly remarkable, resonant, magneto-phonon effect if the Raman scattering response of the $\mathrm{E}_{2 g}$ phonon (so called G-band) is investigated as a function of the magnetic field $B$ applied across the layer. In this case, the two-dimensional (2D) energy bands of graphene condense into discrete Landau levels $\mathrm{L}_{ \pm n}$ with energies $E_{ \pm n}= \pm v_{F} \sqrt{2 e \hbar B n}$ $(n=0,1,2, \ldots)$. Then, the $\mathrm{E}_{2 g}$ phonon hybridizes with the selected $\mathrm{L}_{-n-1(-n)} \rightarrow \mathrm{L}_{n(n+1)}$ inter Landau level (LL) excitations $\frac{1.2}{2}$. As a consequence, a series of avoided crossing events (in clean systems) and/or noticeable magneto-oscillations in the phonon response (in more disordered systems) can be seen in Raman scatter-

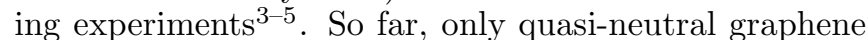
structures; epitaxial graphene $\mathrm{e}^{3}$ and "electronically decoupled" graphene on a graphite surface $\underline{\underline{4}-\underline{6}}$, have shown a clear magneto-phonon resonant effect. The amplitude of this effect depends on the electron-phonon coupling constant $\lambda$ and the oscillator strength of the inter LL transition involved, which includes matrix elements and occupation factors of the initial and final state Landau levels. For neutral graphene, where the Fermi level is pinned to the $\mathrm{L}_{0}$ level, all inter-LL excitations are active. Indeed this system shows a rich series of avoided crossings events each time the $\mathrm{L}_{-n-1(-n)} \rightarrow \mathrm{L}_{n(n+1)}$ transition approaches the $\mathrm{E}_{2 g}$ mode. It is interesting to examine the case of doped graphene where the Fermi energy is far away from the neutrality point $\left(\mathrm{L}_{0}\right.$ level $)$ and a part of the $\mathrm{L}_{-n-1(-n)} \rightarrow \mathrm{L}_{n(n+1)}$ transitions become inactive due to Pauli blocking. The latter implies the suppression of their hybridization with the optical phonon. Polarization resolved Raman scattering experiments are expected to be crucial for tracking the magneto-phonon resonance in doped graphene ${ }^{2}$. Indeed, the two, degener- ate in energy $\mathrm{L}_{-n} \rightarrow \mathrm{L}_{n+1}$ and $\mathrm{L}_{-n-1} \rightarrow \mathrm{L}_{n}$ transitions have identical oscillator strength in neutral graphene but not necessarily so if the Fermi energy is pushed away from the neutrality point. $\mathrm{L}_{-n} \rightarrow \mathrm{L}_{n+1}$ and $\mathrm{L}_{-n-1} \rightarrow \mathrm{L}_{n}$ excitations carry either $\mathrm{m}_{z}=+1$ or -1 angular momentum. Their possible difference in hybridization strength with the $\mathrm{E}_{2 g}$ phonon should be detectable with the circular polarization of the incident and scattered photons ${ }^{2}$.

Here, we report on the progress in searching for a magneto-phonon resonance in monolayer graphene on $\mathrm{Si} / \mathrm{SiO}_{2}$ substrate. We employed the high-field magnetoRaman scattering methods and applied polarization resolved measurements to investigate the graphene's Gband in two relevant, $\sigma^{+} / \sigma^{-}$and $\sigma^{-} / \sigma^{+}$configurations of circularly polarized excitation/scattered light. The $\mathrm{E}_{2 g}$ phonon shows a pronounced hybridization only with the fundamental inter LL transition (involving the $\mathrm{L}_{0^{-}}$ level) at a resonant field $\mathrm{B} \approx 25 \mathrm{~T}$ and, distinctly, only in the case of one of the two polarization configurations. This behavior is shown to be characteristic for graphene with carrier concentration of $\sim 2 \times 10^{12} \mathrm{~cm}^{-2}$, typical for untreated samples of graphene on $\mathrm{Si} / \mathrm{SiO}_{2}$. Such a relatively strong, either n- or p-type, doping implies that all interband transitions involving LLs with indexes $n>1$ are blocked due to the occupation factor. Nevertheless at $\mathrm{B} \sim 25 \mathrm{~T}$ the LL filling factor is $2<v<6$, and either $\mathrm{L}_{0} \rightarrow \mathrm{L}_{1}$ (electron doping) or $\mathrm{L}_{-1} \rightarrow \mathrm{L}_{0}$ (hole doping), but not both of them, become active. This explains our observation of the pronounced "circular dichroism" in the magneto-phonon resonance of doped graphene.

The magneto-Raman scattering experiments have been carried out in back-scattering Faraday geometry using a homemade set-up - see Ref, 6 for details of technical arrangements. The $488 \mathrm{~nm}$ line of an $\mathrm{Ar}^{+}$ion laser has been used as the excitation source ${ }^{7}$. The laser light (with 


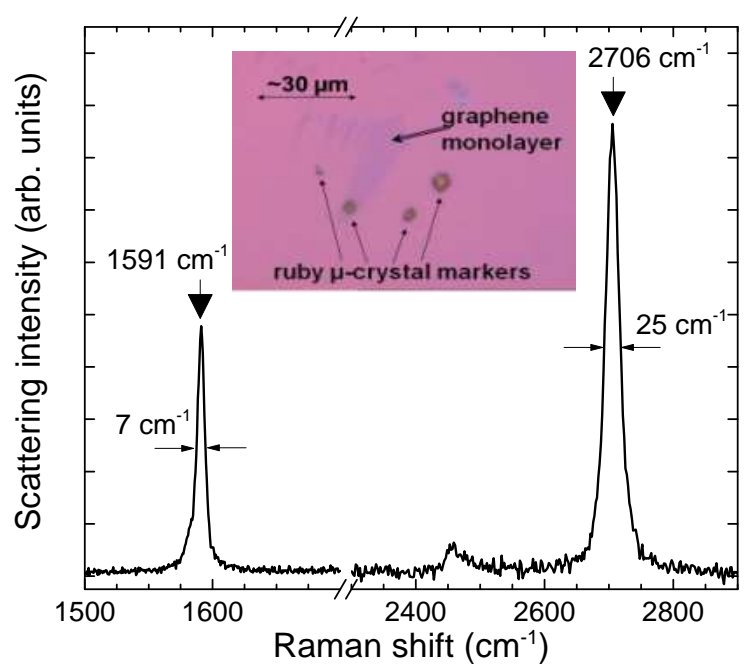

Figure 1: Raman scattering spectra measured at temperature $\mathrm{T}=4.2 \mathrm{~K}$ and under $488 \mathrm{~nm}$ laser line excitation, showing the characteristic $\mathrm{E}_{2 g}$ and $2 \mathrm{D}$ bands of the graphene investigated. The inset shows the optical image of the sample.

power $\sim 1 \mathrm{~mW}$ ) has been focused on the sample, down to a spot of $\sim 1 \mu \mathrm{m}$ in diameter. The sample has been mounted on an XYZ piezo-stage. The measurements, in two distinct, $\sigma^{+} / \sigma^{-}$and $\sigma^{-} / \sigma^{+}$configurations for the circular polarisation of the incident/collected light were realized by inverting the direction of the magnetic field, while keeping fixed the orientation of all optical elements (e.g., polarizers). Experiments have been carried out at liquid helium temperatures $(\mathrm{T}=4.2 \mathrm{~K})$ and in magnetic fields up to $29 \mathrm{~T}$ (supplied by a resistive magnet).

The studied graphene sample was exfoliated from natural graphite and deposited on top of a $\mathrm{Si} / \mathrm{SiO}_{2}$ wafer (oxide thickness of $300 \mathrm{~nm}$ ). Its optical microscope image is shown in the inset of Fig. 1. Micron-size ruby crystals (giving rise to a strong luminescence signal at $\sim 692 \mathrm{~nm}$ ) have been deposited in the vicinity of the graphene flake in order to facilitate the localization of the sample with respect to the laser spot. Raman scattering spectra measured in the absence of the magnetic field and at temperature $\mathrm{T}=4.2 \mathrm{~K}$ confirm the monolayer character of the graphene structure investigated ${ }^{8,9}$ (see Fig. 1). The so-called $2 \mathrm{D}$ band, centered at $2706 \mathrm{~cm}^{-1}$, fairly represents a single Lorentzian line with the characteristic Full Width at Half Maximum, FWHM $=25 \mathrm{~cm}^{-1}$. The Gband shows the characteristic width of FWHM $=7 \mathrm{~cm}^{-1}$ and is centered at $1591 \mathrm{~cm}^{-1}$. Both its small width and energy position point towards a considerable (electron or hole) doping of the investigated graphene sample $\mathrm{e}^{10,11}$.

Our experimental findings are presented in Fig. 2. This figure illustrates the evolution with the magnetic field of the measured magneto-Raman spectra of the $\mathrm{E}_{2 g}$ phonon in two, distinct, $\sigma^{-} / \sigma^{+}$and $\sigma^{+} / \sigma^{-}$configurations of circularly polarized excitation/scattered light. The prominent feature seen in this figure is a pronounced circular magneto-dichroism of the Raman scattering spectra of the investigated G-band. This band is practically unaffected by the magnetic field when probed in one $\sigma^{+} / \sigma^{-}$ polarization configuration. Focusing more on the results obtained in this configuration we find that the FWHM of the G-band does not indeed change with the magnetic field up to the highest values explored, of $29 \mathrm{~T}$. The central position of the G-band shows, however, a small downward (red) shift in energy of up to $\sim 2 \mathrm{~cm}^{-1}$ at $\mathrm{B}=29 \mathrm{~T}$, the shift remaining smaller than the linewidth. The evolution of the G-band with the magnetic field is clearly much richer in the opposite $\sigma^{-} / \sigma^{+}$configuration. In this case, a pronounced blue shift of the spectral weight of the G-band, associated with noticeable spectral broadening, is seen in the spectra in magnetic fields up to $24 \mathrm{~T}$. The shift reaches $20 \mathrm{~cm}^{-1}$ and the spectrum extends over $50 \mathrm{~cm}^{-1}$. At $\mathrm{B} \sim 25 \mathrm{~T}$, the G-band practically disappears from the spectra, likely being smeared over a very broad energy range. At still higher fields, the G-band reappears in the spectra as a broad line but this time on the low energy side of its original position ( at $\mathrm{B}=0$ ). This characteristic evolution of the $\sigma^{-} / \sigma^{+}$component of the G-band points towards its effective hybridization (avoided crossing) with one of the $\mathrm{L}_{-1} \rightarrow \mathrm{L}_{0}$ or $\mathrm{L}_{0} \rightarrow \mathrm{L}_{1}$ transitions. At the same time, none of those two excitations couple with the $\sigma^{+} / \sigma^{-}$component of the G-band. We therefore suspect that we are dealing with strongly doped graphene, such that at high fields around $\mathrm{B}=25 \mathrm{~T}$, the Landau level filling factor is $2<|\nu|<6$. Then, in case of electron doping $(\nu>0)$ the $\mathrm{L}_{0}$ Landau level is fully populated with electrons but $\mathrm{L}_{1}$ is partially empty and $\mathrm{L}_{0} \rightarrow \mathrm{L}_{1}$ transition is active but $\mathrm{L}_{-1} \rightarrow \mathrm{L}_{0}$ is Pauli blocked. In case of hole doping $(\nu<0)$ the $L_{0}$ level is completely empty but electrons occupy the $L_{-1}$ level and then the $\mathrm{L}_{-1} \rightarrow \mathrm{L}_{0}$ transition is active but not $\mathrm{L}_{0} \rightarrow \mathrm{L}_{1}$. In consequence, the $\mathrm{E}_{2 g}$ phonon can couple with only one of possible (fundamental) transitions involving the $L_{0}$ level, which broadly explains the strong circular dichroism observed in our magneto-Raman scattering data.

To ascertain the light helicities of our polarization configurations, we have replaced the graphene sample by a semiconductor, $\mathrm{GaAs} /(\mathrm{Ga}, \mathrm{Al}) \mathrm{As}$ quantum well structure and calibrated the experimental set-up by measuring the near band-edge magneto-luminescence of this structure with a well known sequence of two, Zeeman-split, polarization-resolved components $\frac{12}{2}$. Taking into account the characteristic for graphene selection rules 2.13 resulting from the transfer of angular momentum we deduce that in the $\sigma^{-} / \sigma^{+}$configuration the $\mathrm{E}_{2 g}$ phonon couples to the $\mathrm{L}_{-1} \rightarrow \mathrm{L}_{0}$ transition and therefore conclude that our graphene is hole doped. An initial inspection of the data points at the resonant field, when $E_{2 g}=E_{1}$, of $B \simeq 25 T$. This is somewhat lower as compared to previous observations, at $\mathrm{B} \simeq 28 \mathrm{~T}$ in case of quasi neutral epitaxial graphene $e^{14.15}$ and graphene on graphite $\frac{4.5}{1}$, and indicates a slightly higher Fermi velocity $\left(\simeq 1.1 \times 10^{6} \mathrm{~m} / \mathrm{s}\right.$ instead of $\left.\simeq 1.0 \times 10^{6} \mathrm{~m} / \mathrm{s}\right)$ in the present case of graphene flake on $\mathrm{Si} / \mathrm{SiO}_{2} \frac{16,17}{2}$. We use this value of Fermi veloc- 


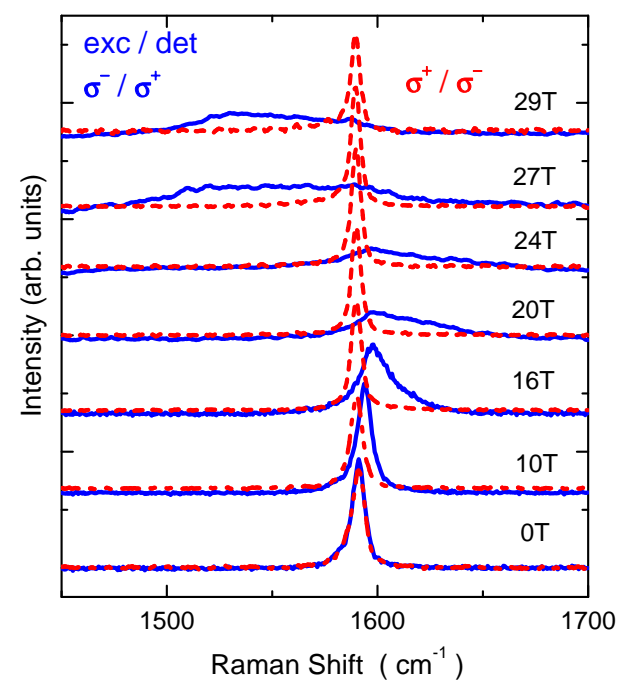

Figure 2: (Color online) Typical Raman scattering spectra of the $\mathrm{G}$ band feature in the two crossed circular polarization configurations (dashed line corresponds to $\sigma^{+} / \sigma^{-}$) at various values of the magnetic field. Spectra are shifted for clarity.

ity in our simulations. We speculate that different "effective" Fermi velocities in different samples might be also accounted for by different degree of disorder but a discussion of those effects is beyond the scope of this paper and will be presented elsewhere.

With the aim to better support our data interpretation, we illustrate in Fig.3 the three representative regimes (with respect to carrier concentration) of the magneto-phonon resonance in graphene. Hole doping is considered, however, the results hold for n-type graphene as well but with the inverted polarization configurations. Following the concepts developed in Refs 1,2 we refer to the phonon Green function :

$$
\tilde{\epsilon}^{2}-\epsilon_{0}^{2}=2 \epsilon_{0} \lambda E_{1}^{2} \sum_{k=0}^{\infty}\left\{\frac{f_{k} T_{k}}{(\tilde{\epsilon}+i \delta)^{2}-T_{k}^{2}}+\frac{1}{T_{k}}\right\}
$$

which defines the actual phonon energy $\epsilon=\operatorname{Re}(\tilde{\epsilon})$ (and broadening $\operatorname{Im}(\tilde{\epsilon})$ ), and in which $\epsilon_{0}$ is the phonon energy of the neutral system at $B=0 . \quad \delta$ accounts for the broadening characteristic for electronic transitions. $T_{k}=(\sqrt{k}+\sqrt{k+1}) E_{1},(k=0,1,2, \ldots)$, stands for energies of inter-Landau level excitations which possibly couple to the phonon. $f_{k}$, which varies from 0 to 1 , is defined as the filling factor of the single Landau level and accounts for the occupancy of the initial and final states involved in a given $\mathrm{L}_{-n-1(-n)} \rightarrow \mathrm{L}_{n(n+1)}$ excitation.

In the following, we consider only the phonon energy $\epsilon$, and assume the same broadening of electronic states of $\delta=90 \mathrm{~cm}^{-1}$ as in Ref. 3 which partially smears the effect of mode hybridization (jumps in energy rather than clear anticrossings). The phonon energy in two different crossed polarization configurations is calculated by two separate solutions of Eq.1 : the first taking into account only the $\Delta|n|=+1$ inter Landau transitions $\left(\mathrm{L}_{-n} \rightarrow \mathrm{L}_{n+1}\right)$ and the second one accounting separately for $\Delta|n|=-1$ excitations $\left(\mathrm{L}_{-n-1} \rightarrow \mathrm{L}_{n}\right)$.

Varying the carrier concentration, one may distinguish three distinct regimes for the magneto-phonon resonance in graphene. The first one appears at relatively low doping, when $E_{2 g}>2\left|E_{F 0}\right|$ ( $E_{F 0}$ stands for the Fermi energy at zero magnetic field). Then, practically all $\mathrm{L}_{-n-1(-n)} \rightarrow \mathrm{L}_{n(n+1)}$ transitions are active and effectively hybridize with the phonon (see two upper panels of Fig. 3). In this case the nonvanishing carrier concentration results in a rather small asymmetry in the two distinct polarization configurations, the details of the asymmetry being determined by the actual evolution of the Fermi energy with the magnetic field. The second regime is illustrated in two middle panels of Fig. 3 . It reflects the situation when $\sqrt{2}\left|E_{F 0}\right|$ exceeds $\mathrm{E}_{2 g}$ and that all $\mathrm{L}_{-n-1(-n+1)} \rightarrow \mathrm{L}_{n(n)}$ transitions with $n \geq 1$ are Pauli blocked at magnetic fields of their resonances with the phonon. At the same time, the carrier concentration can be low enough that at the field value when $E_{1}=E_{2 g}$, the Fermi energy is pinned to the $\mathrm{L}_{-1}$ level $\left(2\left|E_{F 0}\right|<\sqrt{6} E_{2 g}\right)$ and therefore the $\mathrm{L}_{-1} \rightarrow \mathrm{L}_{0}$ transition is active and it resonantly hybridizes with the phonon. Notably this is the only resonant hybridization event possible in this case which likely corresponds to the present experimental results.

Apart of the pronounced anticrossing events, the $E_{2 g}$ phonon is also affected by interactions with other, more distant in energy, inter LL excitations. This leads to an additional, though much weaker and rather smooth evolution of the G-band with the magnetic field. These nonresonant effects persist even in case of high carrier concentrations where they appear to be particularly transparent and can be estimated analytically. We discuss them here in an attempt to account for the small red shift of the phonon energy with the magnetic field, which is observed in the inactive $\sigma^{+} / \sigma^{-}$polarisation configuration. As illustrated in the bottom panels of Fig. 3, when the hole concentration is high enough, i.e., when $\left(2\left|E_{F 0}\right|>\sqrt{6} E_{2 g}\right)$, all resonant hybridizations of the $\mathrm{E}_{2 g}$ phonon with $\mathrm{L}_{-n-1(-n)} \rightarrow \mathrm{L}_{n(n+1)}$ excitations are quenched. Nevertheless, the G-band still displays certain field dependence (and circular dichroism). To elucidate this behavior, we firstly note that the non resonant renormalization of the phonon energy which results from interactions with all high energy $\Delta|n|=+1$ inter Landau level transitions is not the same as the renormalization resulting from the interaction with all $\Delta|n|=-1$ excitations. To see this, imagine that the Fermi level is located low in the band, in between $L_{-l}$ and $L_{-l+1}$ level. Then, there is clearly one more active transition $\left(\mathrm{L}_{-l} \rightarrow \mathrm{L}_{l-1}\right)$ in the series of $\mathrm{L}_{-n} \rightarrow \mathrm{L}_{n-1}$ transitions as compared to the series of $\mathrm{L}_{-n} \rightarrow \mathrm{L}_{n+1}\left(\mathrm{~L}_{-l+1} \rightarrow \mathrm{L}_{l}\right.$ is blocked). Due to this effect, the estimated splitting of the phonon energy (in two different polarization configurations) is $\epsilon_{-}-\epsilon_{+}=\lambda E_{C R}$ where $E_{C R}=v_{F} e B / \sqrt{\pi n_{h}}=$ 


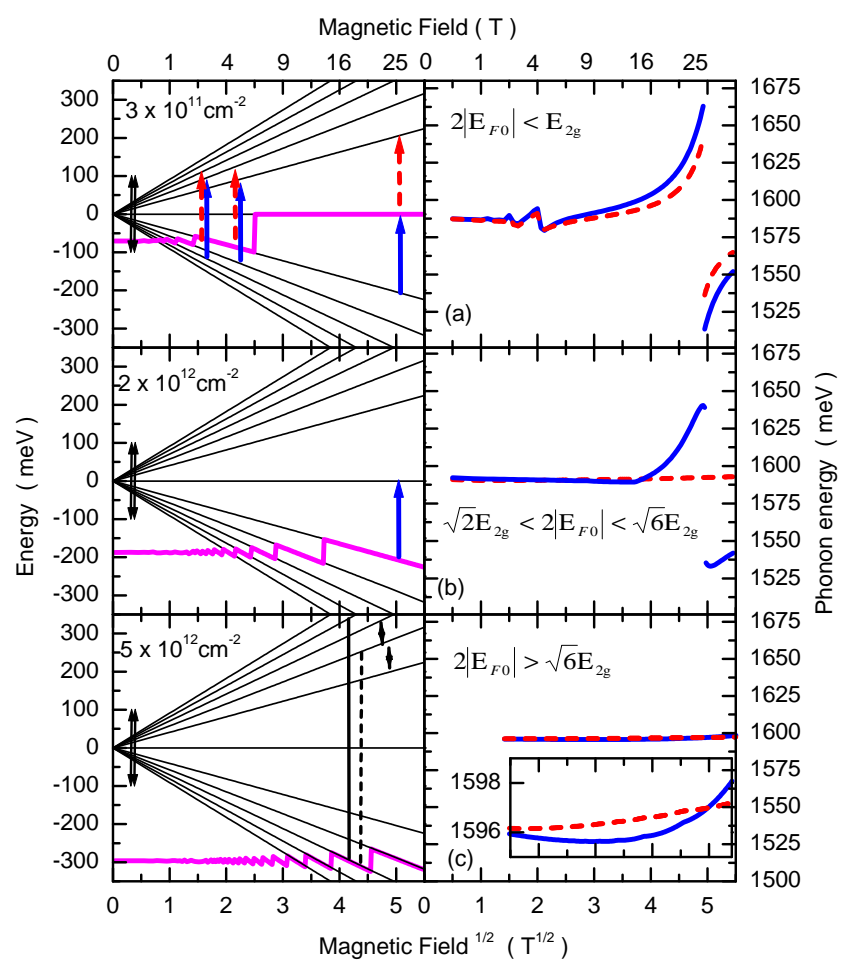

Figure 3: (Color online) Three regimes of the hole gas concentration and its impact on the magneto-phonon effect. Left panels represent fan charts of the Landau levels with marked excitations being in resonance with $E_{2 g}$ phonon (panels a and b). The solid arrows represent excitations in $\sigma^{-} / \sigma^{+}$polarization, dashed in $\sigma^{+} / \sigma^{-}$polarization. The thick line shows the Fermi level. Arrows on panel c represent non resonant excitations (inter- and intra-band). Right panels show the evolution of the phonon energy versus magnetic field, with the same representation of polarizations by solid/ dashed lines.

$E_{1}^{2} /\left(2\left|E_{F 0}\right|\right)$. Secondly, we have so far neglected a possible coupling of the phonon with the (low energy) cyclotron resonance like mode which occurs between neighboring Landau levels in the vicinity of the Fermi level. Notably, this mode, present only in one polarisation, may also couple to the phonon ${ }^{1}$, leading to the estimated blue shift of the phonon energy of about $\lambda \frac{2 v_{F}^{3} \hbar e^{2} B^{2}}{\epsilon_{0}^{2} \sqrt{\pi n_{h}}}$, in the range of magnetic fields considered. Interestingly, an additional resonant hybridization event which involves this cyclotron resonance mode $\frac{18,19}{19}$ may be characteristic of strongly doped graphene but arising at fields much above the range explored in these experiments, i.e., at $B=E_{2 g} \sqrt{\pi n_{h}} / v_{F}$ e if $\nu=\sqrt{n_{h} / \pi} h v_{F} / E_{2 g} \gg 1$. For example for carrier density $n=10^{13} \mathrm{~cm}^{-2}$ the resonance would occur at $\mathrm{B} \sim 110 \mathrm{~T}$. The results of numerical calculations of phonon energies in two polarization configurations for graphene with high hole concentration $\left(n_{h}=5 \times 10^{12} \mathrm{~cm}^{-1}\right)$ are shown in the lowest panel of Fig. 3. The non resonant effects are overall weak but nonetheless the expected trends are against the observation of the noticeable red shift of the phonon experienced

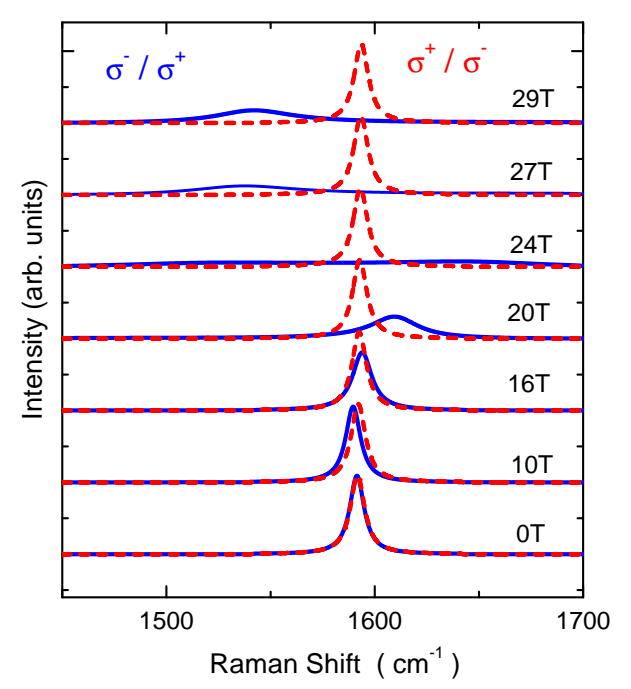

Figure 4: Simulation of the Raman spectra versus magnetic field in two crossed circular polarization configurations for different values of the magnetic field.

in the "inactive" $\sigma^{+} / \sigma^{-}$configuration. This observation remain a puzzle and requires more elaborated theoretical models, perhaps including the effects of possible electron hole asymmetry of the graphene bands and or invoking different coupling mechanism with the cyclotron resonance mode.

The above analysis was done in the ideal case of a well defined, uniform carrier density and for a graphene system with no other scattering than the electronic scattering introduced through the parameter $\delta$ in Eq. 1. Despite their simplicity our simulations help us to clarify the main expected features of the magneto-phonon resonances in graphene in different possible regimes of carrier concentration. The spectra presented in Fig. 2 show no evolution of the phonon feature in one polarization configuration and a clear anti-crossing behavior at $B=25 T$ in the other polarization. This observation indicates that $\mid E_{F 0 \mid}>\sqrt{2} E_{2 g} / 2\left(n_{h}=1.2 \times 10^{12} \mathrm{~cm}^{-2}\right)$ and $\left|E_{F 0}\right|<\sqrt{6} E_{2 g} / 2\left(n_{h}=3.5 \times 10^{12} \mathrm{~cm}^{-2}\right)$. Hence, we are investigating the second density regime, for which a single inter-band electronic excitation, i.e., the $\mathrm{L}_{-1} \rightarrow \mathrm{L}_{0}$ transition is active when tuned in resonance to the phonon energy which results in the observed anticrossing effect.

The qualitative attempt to simulate the shape of the measured spectra is presented in Fig. 4. The simulations imply the calculation of the phonon spectral function, $-\operatorname{Im} D(q, \omega)$, following the procedure described by Ando $\frac{1}{1}$ (Eqs. 2.21 and 3.2) complemented with the associated polarization selection rules $^{2}$. We have set the parameter $\lambda$ to $4.5 \times 10^{-3}$ in line with its value derived in Ref. [3]. Each spectrum is convoluted with a Gaussian function with a FWHM of $6.6 \mathrm{~cm}^{-1}$, in order to reproduce the experimentally observed FWHM of the G-band at zero magnetic field. The parameter $\delta=90 \mathrm{~cm}^{-1}$ and the carrier density $n_{e l}=2 \times 10^{12} \mathrm{~cm}^{-2}$ were adjusted 
to fairly reproduce the experimental shift of the phonon feature in the range of magnetic fields prior to the anticrossing event observed in $\sigma^{-} / \sigma^{+}$polarization configuration. The assumed value of the hole concentration implies a complete quenching of the anticrossing event in the opposite polarization configuration. As mentioned before, the amplitude of the phonon energy shift strongly depends on $\lambda$ - a hypothesis of a smaller value for $\lambda$ would imply an assumption of a smaller value for $\delta$.

With the assumed values of relevant parameters, the main experimental features are fairly well simulated: the phonon feature is almost unchanged up to $29 \mathrm{~T}$ in the $\sigma^{+} / \sigma^{-}$polarization, while in the $\sigma^{-} / \sigma^{+}$configuration the pronounced anticrossing effect, at $\mathrm{B} \simeq 25 \mathrm{~T}$ is reproduced. The main differences between simulation and the experiment are: (i) the smooth development of the blue shift without any distinguishable kink, and (ii) the asymmetric line-shape observed in the experiment close to the resonant magnetic field. These discrepancies, we believe are due to our simplified approach in accounting for the effects of disorder ${ }^{20}$. A more detailed analysis would require taking into account the fluctuations of local carrier density and possibly an exact form of Landau level broadening. Nevertheless we conclude that the experimental observations are overall well reproduced by our calculated spectra.
To conclude, by using two different crossed circular polarization configurations, we have been able to investigate the coupling of the $\mathrm{E}_{2 g}$ phonon either to $\Delta|n|=+1$ or $\Delta|n|=-1$ inter Landau level excitations. The strong anisotropy in the magneto-phonon effect observed in two distinct polarization configurations at high magnetic field is accounted for by relatively high doping of the investigated graphene. The hybridization of the $\mathrm{E}_{2 g}$ phonon is effective with the $\mathrm{L}_{-1} \rightarrow \mathrm{L}_{0}$ transition whereas Pauli blocking of the $\mathrm{L}_{0} \rightarrow \mathrm{L}_{1}$ transition quenches the phonon coupling with this latter electronic excitation. More investigations, in particular on gated graphene structures are needed to uncover all the subtleties of the magnetophonon resonance in graphene.

\section{Acknowledgments}

Part of this work has been supported by GACR P204/10/1020, GRA/10/E006 (EPIGRAT), RTRA DISPOGRAPH projects and by EuroMagNET II under the EU contract number 228043. One of us (PK) acknowledges the support from European Project No. FP7/20072013-221515 (MOCNA).
* Electronic address: Piotr.Kossacki@fuw.edu.pl

1 T. Ando, J. Phys. Soc.Jpn. 76, 024712 (2007).

2 M. O. Goerbig, J. N. Fuchs, K. Kechedzhi and V. Fal'ko, Phys. Rev. Lett. 99, 087402 (2007).

3 C. Faugeras, M. Amado, P. Kossacki, M. Orlita, M. Sprinkle, C. Berger, W. A. de Heer and M.Potemski, Phys. Rev. Lett. 103, 186803 (2009).

4 J. Yan, S. Goler, T. D. Rhone, M. Han, R. He, P. Kim, V. Pellegrini and A. Pinczuk, Phys. Rev. Lett. 105, 227401 (2010).

5 C. Faugeras, M. Amado, P. Kossacki, M. Orlita, M. Kühne, A.A.L. Nicolet, Yu.I. Latyshev and M.Potemski, Phys. Rev. Lett. 107, 036807 (2011).

${ }^{6}$ M. Kühne, C. Faugeras, P. Kossacki, A. A. L. Nicolet, M. Orlita, Yu. I. Latyshev and M. Potemski, Phys. Rev. B 85, 195406 (2012).

7 Y.Y. Wang, Z.H. Ni, Z.X. Shen, H.M. Wang and Y.H. Wu, Appl. Phys. Lett. 92, 043121 (2008).

8 A. C. Ferrari, J. C. Meyer, V. Scardaci, C. Casiraghi, M. Lazzeri, F. Mauri, S. Piscanec, D. Jiang, K. S. Novoselov, S. Roth, A. K. Geim, Phys. Rev. Lett. 97, 187401 (2006).

9 D. Graf, F. Molitor, K. Ensslin, C. Stampfer, A. Jungen, C. Hierold and L. Wirtz, Nano Lett. 7, 238 (2007).

10 J. Yan, Y. Zhang, P. Kim and A. Pinczuk, Phys. Rev. Lett. 98, 166802 (2007).

11 S. Pisana, M. Lazzeri, C. Casiraghi, K. S. Novoselov, A.
K. Geim, A. C. Ferrari and F. Mauri, Nature Mat. 6, 198 (2007).

12 M. Potemski, Physica B 256, 283 (1998).

13 P. Kossacki, C. Faugeras, M. Kühne, M. Orlita, A. A. L. Nicolet, J. M. Schneider, D. M. Basko, Yu. I. Latyshev and and M. Potemski, Phys. Rev. B 84, 235138 (2011).

14 M. L. Sadowski, G. Martinez, M. Potemski, C. Berger and W. A. de Heer, Phys. Rev. Lett. 97, 266405 (2006).

15 M. Orlita, C. Faugeras, P. Plochocka, P. Neugebauer, G. Martinez, D. K. Maude, A. -L. Barra, M. Sprinkle, C. Berger, W. A. de Heer and M. Potemski, Phys. Rev. Lett. 101, 267601 (2008).

${ }^{16}$ Z. Jiang, E. A. Henriksen, L. C. Tung, Y. J. Wang, M. E. Schwartz, M. Y. Han, P. Kim and H. L. Stormer, Phys. Rev. Lett. 98, 197403 (2007).

17 R.S. Deacon, K.C. Chuang, R.J. Nicholas, K.S. Novoselov and A.K. Geim , Phys. Rev. B 76, 081406(R) (2007).

18 A.M. Witowski, M. Orlita, R. Stepniewski, A. Wysmolek, J.M. Baranowski, W. Strupinski, C. Faugeras, G. Martinez and M. Potemski, Phys. Rev. B 82, 165305 (2010).

19 M. Orlita, I. Crassee, C. Faugeras, A.B. Kuzmenko, F. Fromm, M. Ostler, T. Seyller, G. Martinez, M. Polini and M. Potemski, New Journal of Physics 14, 095008 (2012).

20 O. Kashuba and V.I. Fal'ko, New Journal of Physics 14, 105016 (2012). 